\title{
An analysis of energy storage system interaction in a multi objective model predictive control based energy management in DC microgrid
}

\author{
Unnikrishnan Raveendran Nair, Ramon Costa-Castelló \\ Institut de Robòtica i Informòtica industrial,CSIC-UPC \\ Llorens i Artigas 4-6, 08028 Barcelona, Spain \\ uraveendran@iri.upc.edu,ramon.costa@upc.edu
}

\begin{abstract}
Non-deterministic generation from renewable sources have resulted in the incorporation energy storage systems in modern grids. Management of energy between different storage elements need to done optimally to ensure efficient operation of the grid. The intraday energy management problem is addressed in this work through an online model predictive control using multi objective optimisation. This work analyses the energy interaction among different storages when penalty weights in a multi objective optimisation problem is varied, in order to find an optimal scenario in terms of weight distribution. Different scenarios are identified and performance indices are proposed to achieve the same. The work also addresses implicitly the objective of minimising rate of degradation batteries. Simulation results are presented to aid in the analysis.
\end{abstract}

Index Terms-model predictive control, energy management, energy storages system, degradation rate

\section{INTRODUCTION}

The increased penetration of renewable source has resulted in a shift from dispatchable to non-dispatchable, nondeterministic generation. This comes with associated problems of ensuring grid stability, power quality and supply demand balance in the grid. These issues are alleviated through the incorporation of energy storage system (ESS) which perform multitude of functions like grid support, power quality improvement, ensuring power balance, demand side management to name a few [1], [2]

The control of grid/microgrid is carried out in a hierarchical fashion differentiated mainly by sampling time period of each level and dynamics each level addresses. This is shown in Fig.1. This hierarchical control can be divided into two

This work is done as part of project which has received funding from the European Unions Horizon 2020 research and innovation programme under the Marie Skodowska Curie grant agreement No 675318 (INCITE). This work has been partially funded by the Spanish national project MICAPEM (ref. DPI2015- 69286-C3-2-R, MINECO/FEDER). This work is supported by the Spanish State Research Agency through the Mara de Maeztu Seal of Excellence to IRI (MDM-2016-0656). This work is partially funded by AGAUR of Generalitat de Catalunya through the Advanced Control Systems (SAC) group grant (2017 SGR 482). This work has been done with the support of ACCI (Operational Program FEDER Catalunya 2014-2020) through the REFER project (COMRDI15-1-0036-11). general parts namely: short term power balancing and long term energy management [3]. The short term power balancing side of the control is mainly tasked with maintaining the system stability, voltage and frequency regulation (AC grids) under disturbances arising from unforeseen load, generation variations. As the name suggests this level ensures power balance in the grid at every instance thereby ensuring stability. The controller in this level address dynamics in the range of seconds to milliseconds which require use of fast action and robust controllers. In the long term energy management level the controller objective is to manage the energy distribution among the different elements in grid such that some operating parameter is optimised. This level also use historical generation and load data to predict future scenarios which aids in the effective decision making of energy management. This level is characterised by longer sampling time and slower controller dynamics [4], [5]. The hierarchical structure of control is also characterised by flow of system information and control action in the directions shown in Fig.1 [3].

The ESS used in grid connected applications are classified into two categories depending on the physical attributes of the ESS. They are categorised as high power and high energy density ESS. The high power density ESS include supercapacitors (SC), flywheels, etc. which are capable of handling fast power dynamics (sudden power changes). This enables them to be used in applications like ensuring grid power balance, grid power quality control, voltage regulation etc. Therefore these type of ESS are mostly employed in and controlled by the short term power balance level. These ESS cannot store large amount of energy. The second group of ESS are characterised by their high energy density. They are capable of storing large amounts of energy but cannot cater to sudden power variations. As such they find increased application in the long term energy management level which also controls them. These include batteries, fuel cell (FC)- electrolyser system, pumped hydro storages to name a few [1], [6], [7]. In microgrid application employing heterogeneous ESS will ensure efficient operation and optimal sizing of the storage systems [8].

The energy management in heterogeneous ESS based sys- 


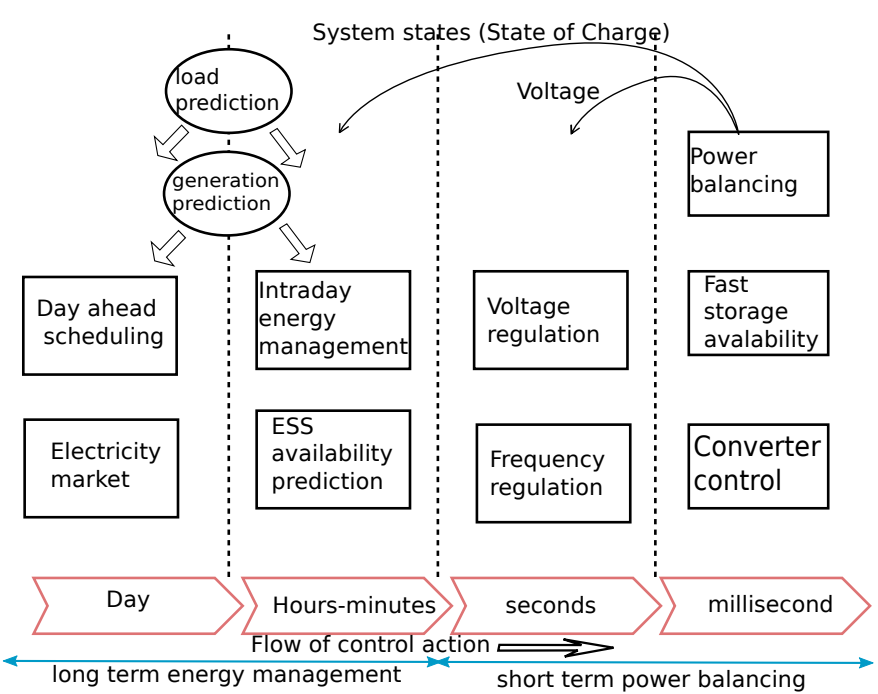

Fig. 1. Control architecture for renewable energy based microgrid employing ESS

tem has been addressed using heuristic rule based or fuzzy inference based techniques [9]-[11], offline optimal control [12]-[14] and online model predictive control (MPC) based techniques [15]-[17]. The offline control mainly deals with the day ahead decision making for optimal resource allocation as shown in Fig.1. The MPC based techniques, on the other hand, deals with the intraday energy management problem based on real time system states and are implemented as an online control.

In this paper the intraday control using MPC techniques for energy management among the ESS is analysed. The offline day ahead scheduling stage is not considered in this problem. The objective is to study the interaction among different ESS in a heterogeneous storage system when weights of the multi objective optimisation problem is varied. The cycling of energy, overcharging and deep discharge in different ESS will be analysed under various scenarios to identify near optimal weight distribution. As far as the authors knowledge goes such an analysis was not carried out before as most of the previous work focussed on proposing improved control techniques [9]-[17]. This will help to identify a general consensus on how the weights should be distributed when the objectives in a multi-objective problem tend to complimentary. In this case the objectives considered are degradation rate of ESS and efficiency of operation. Apart from this the authors also propose an indirect method to minimise the degradation rates of batteries based on the ideas of non linear degradation models presented in [18]-[20]. The proposal aims to keep the optimisation problem quadratic which enables easiness in solving. The proposed energy management will be for a DC microgrid.

The rest of the paper is organised as follows. In section II the considered system and its models for the intraday energy management using MPC is presented. In section III the optimisation problem is formalised. In section IV the results of

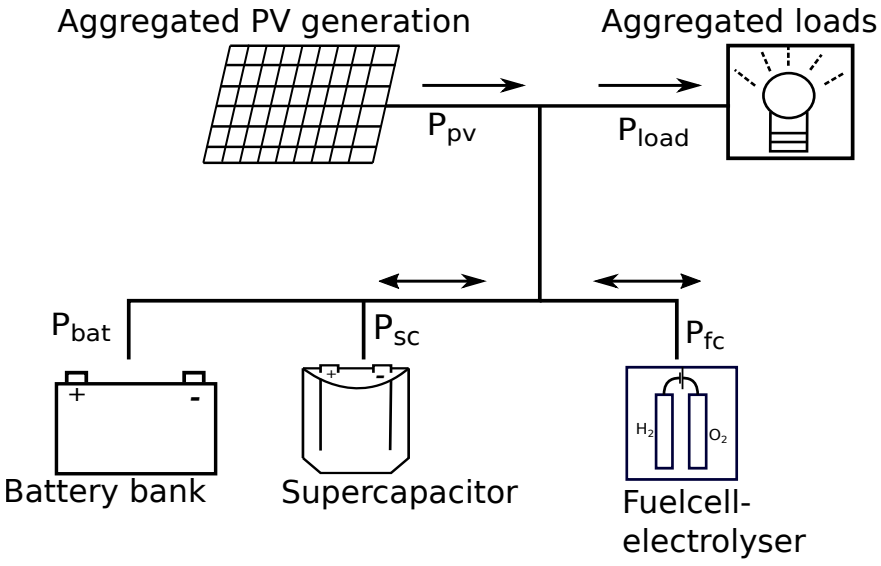

Fig. 2. Schematic of the proposed microgrid under consideration

MPC control is presented in simulation along with a study of different cases where the weights of objective functions (from MPC) are varied. Some performance indices are proposed for each case to enable an easy comparison. Results of low level control performance through co-simulation is also presented. Finally is section $\mathrm{V}$ the work is concluded

\section{SYSTEM DESCRIPTION}

The DC microgrid under consideration for the unit commitment problem is shown in Fig.2. It represents an aggregated system with generation from PV panels, energy storage systems (ESS) in the form of batteries, fuelcell-electrolyser system, SC which are all catering to the loads. In the proposed work, autonomous operation of the microgrid under islanded mode is considered. It assumed that the energy management system has access to generation, load profiles resulting in a deterministic system. In order to develop the intraday energy management system the first step will to present the models of ESS and the grid.

\section{A. ESS model}

The ESS are modelled using discrete-time state equations showing the evolution of energy stored in them during intraday operation. In the case of batteries and supercapacitors (SC) they are represented by hybrid equations showing state of charge (SOC) evolution given by

$$
\begin{aligned}
S O C_{\alpha}(i+1) & = \begin{cases}S O C_{\alpha}(i)-\frac{T_{s} \cdot \eta_{\alpha} \cdot}{C_{\alpha}} \cdot p_{\alpha}(i) & \text { if } p_{\alpha} \leq 0 \\
S O C_{\alpha}(i)-\frac{T_{s} \cdot}{\eta_{\alpha} \cdot C_{\alpha}} \cdot p_{\alpha}(i) & \text { if } p_{\alpha}>0\end{cases} \\
\forall \alpha & =\{b a t, s c\}
\end{aligned}
$$

where $S O C_{\alpha}(i)$ represents the SOC of ESS considered at $i_{t h}$ instant, $T_{s}$ is the sample period, $p_{\alpha}(i)$ is the power set point, $C_{\alpha}$ is the capacity of respective ESS and $\eta_{\alpha}$ is the efficiency of power converter interfacing the respective ESS to grid. 
In the case of FC, energy storage is in the form of hydrogen and hence the model is presented by the state of hydrogen (SOH) evolution given by

$S O H_{f c}(i+1)= \begin{cases}S O H_{f c}(i)-\frac{T_{s} \cdot \eta_{f c}}{C_{f c}} \cdot p_{f c}(i) & \text { if } p_{f c} \leq 0 \\ S O H_{f c}(i)-\frac{T_{s} .}{\eta_{f c} \cdot C_{f c}} \cdot p_{f c}(i) & \text { if } p_{f c}>0\end{cases}$

where $p_{f c}, C_{f c}$ are the power set point and the storage capacity of fuel cell respectively. The above model encompasses a FC- electrolyser system which uses electrolyser for generating hydrogen from surplus energy and FC to supply deficient energy. The converter dynamics are not considered in the ESS model as they are in the range of milliseconds

\section{B. Grid model}

In intraday problem considered here the grid is represented as a static system given in discrete-time by the power balance equation

$$
p_{\text {sc }}(i)+p_{\text {bat }}(i)+p_{F C}(i)+p_{\text {ren }}(i)+p_{\text {load }}(i)=0
$$

where $p_{\text {bat }}(i), p_{s c}(i), p_{F C}(i)$ are the same the ESS powers while $p_{\text {ren }}(i), p_{\text {load }}(i)$ are the power generated by the renewable sources and load demand respectively at any sampling instant.

\section{ENERGY MANAGEMENT PROBLEM}

As mentioned before the objective of intraday energy management problem is the distribution of imbalance power in the grid among ESS such that degradation of ESS and grid operation efficiency is optimised. In this context a receding horizon model predictive control (MPC) was adopted for the problem. The MPC controller samples the system and solves an optimisation problem using the system models defined above for a future horizon using the sampled states as initial conditions. The result of this optimisation problem will sequence of set points for the ESS. Thereafter, the first set point from the sequence is applied to the system and this process is repeated [21].

In order, to formulate the MPC optimisation problem cost functions and system operating constraints need to be defined first. These are defined such that they always form a QP (quadratic programming) problem such that they can easily be solved online and solution is guaranteed. The formulations aims at proposing problems where the solution time is small enough that they can integrated into an online controller.

\section{A. Cost function}

The multi objective cost function considered for the problem is given by

$$
J(i)=\sum_{i=1}^{N-1}\left(J_{b a t}(i)+J_{f c}(i)+J_{s c}(i)\right)
$$

where $J_{b a t}, J_{f c}, J_{s c}$ are the costs associated with the battery, FC and SC at the ith sampling instant which will be discussed in details in subsequent sections and $\mathrm{N}$ is the prediction horizon window length. As mentioned in the introduction each $J_{b a t}, J_{f c}$ will have one part dedicated to optimising operating efficiency and another for minimising rate of degradation.

1) Battery cost function: The battery cost $J_{b a t}$ is given by

$J_{b a t}=\sum_{i=1}^{N-1} c_{b a t} \cdot{\frac{p_{b a t}(i)^{2}}{p_{b a t}^{\text {max }}}}^{2}+c_{s o c} \cdot S O C_{b a t}(i)^{2}+c_{d o d} \cdot D O D_{b a t}(i)^{2}$

where $c_{b a t}, c_{s o c}, c_{d o d}$ are the respective weights of each term in $J_{b a t}$, while $S O C_{b a t}, D O D_{b a t}, p_{b a t}^{\max }$ are the state of charge, depth of discharge (DOD) and maximum power of battery respectively. The DOD of any ESS is defined as $\left(D O D_{e s s}=\right.$ $\left.1-S O C_{e s s}\right)$. The different terms are chosen so as to improve efficiency of battery operation and minimise the rate of degradation. The cycle efficiencies of batteries are always less than 100 percent and depend on the type of batteries. Li-ion battery have a cycle efficiency greater than 90 percent [6]. The penalising of normalised battery power in (5) aims to improve the efficiency of operation

In the case of degradation batteries are subjected to calender life and electrochemical ageing. The latter is attributed mainly to operating principle of the battery. Degradation in form of capacity fade arises from changes in electrode-electrolyte interface, change in active material and composite electrode. The main factors responsible are operating conditions, high cycling rates, high SOC and high C-rate discharges [22]-[24]. The battery cost function in (5) tries to minimise degradation by penalising high state of charge and deep discharges (DOD). It also tries to address the issues related to battery cycle. In the autonomous microgrid operation its impossible to eliminate cycling of battery. Nevertheless the cycle magnitude can be minimised. The dependency of degradation to cycle magnitudes have been quantified in [18]-[20] but with non linear formulations. This makes an explicit implementation of these function in optimisation problem difficult to solve and ensure an optimal solution. In this work this issue is circumvented by penalising both high SOC and DOD thereby limiting cycle magnitudes. this helps to retain the quadratic formulation of the cost function which can be easily solved using commercial solvers.

2) Fuel cell cost function: The fuel cell cost function $J_{f} c$ is given by

$$
J_{f c}=\sum_{i=1}^{N-1} c_{f c} \cdot{\frac{p_{f c}(i)^{2}}{p_{b f c}^{\text {max }}}}^{2}+c_{\text {rate }} \cdot\left(p_{f c}(i+1)-p_{f c}(i)\right)^{2}
$$

where $c_{f c}, c_{\text {rate }}$ are the respective weights of each term in $J_{f c}, p_{f c}^{\max }$ is the maximum power that can be delivered by FC. The objective as with the case of battery is to optimise the operational efficiency and minimise degradation. The penalising of normalised battery power aids in improving the operational efficiency. In the case of fuel cell a prominent cause for degradation is the fuel starvation induced due to sudden changes in load demanded from the fuel cell. The effect of fuel starvation on the physical properties of the fuel cell has 
been extensively studied in [25]-[28]. A such penalising the sudden changes in FC set points in (7) can help reduce the rate of degradation.

3) Supercapacitor cost function: The cost function for the supercapacitor is given by

$$
J_{s c}=\sum_{i=1}^{N-1} c_{s c}\left(S O C_{s c}(i)-S O C_{n o m}\right)^{2}
$$

where $c_{s c}$ is the associated weight on the $\mathrm{SC}$ cost function. The role of SC is to act as buffer storage for sudden power changes to maintain the grid voltage within prescribed limits. The voltage regulation control handles the power set points for SC. The SC is included in the MPC to ensure that it has sufficient ability to absorb or give surplus and deficient powers. To this extent the $J_{s c}$ penalises the SC SOC deviation from a pre defined nominal value $\left(S O C_{n o m}\right)$. There is no penalising for the SC power as they have high cycle life and efficiency arising mainly from its operational principle [29].

\section{B. Constraints}

The optimisation problem should address the constraints arising from operating, physical limits and electrical characteristics of associated power interfaces used. The constraints on physical limit of ESS is introduced as the bounds on SOC and $\mathrm{SOH}$. These are represented as

$$
\begin{aligned}
S O C_{\alpha}^{l} & \leq S O C_{\alpha}(i) \leq\left. S O C_{\alpha}^{u}\right|_{\alpha=\{b a t, s c\}} \\
S O H_{f c}^{l} & \leq S O H_{f c}(i) \leq S O H_{f c}^{u}
\end{aligned}
$$

where $S O C_{\alpha}^{l}$ is the lower bound and $S O C_{\alpha}^{u}$ is the upper bound on SOC of battery or supercapacitor, while $S O H_{f c}^{l}, S O H_{f c}^{u}$ are the lower and upper bounds on the hydrogen storage capabilities for FC.

It should be noted that introducing hard constraints as in (8) can lead to controller failing due to system being driven out of feasible region by disturbance or dynamics which are not modelled from microgrid operation. Therefore it is essential to define (8) using soft constraints which allows the deviation from bounds but with heavy penalisation. In the feasible region there is no penalty. This is achieved through the use of slack variables [30] resulting in (8) reformulated as

$$
\begin{aligned}
S O C_{\alpha}^{l}-\epsilon_{\alpha} & \leq S O C_{\alpha}(i) \leq S O C_{\alpha}^{u}+\left.\epsilon_{\alpha}\right|_{\alpha=\{b a t, s c\}} \\
S O H_{f c}^{l}-\epsilon_{f c} & \leq S O H_{f c}(i) \leq S O H_{f c}^{u}+\epsilon_{f c}
\end{aligned}
$$

Constraints are also imposed on the power that can be delivered by the ESS. These are decided based on the electrical operation limits of the ESS and characteristics like current rating, efficiency of the associated power electronic interface. Imposing bounds on power delivered will also ensure that high C-rate discharges do not occur. These are given by

$$
p_{\alpha}^{\min } \leq p_{b a t}(i) \leq\left. p_{\alpha}^{\max }\right|_{\alpha=\{b a t, s c, f c\}}
$$

where $p_{\alpha}^{\min }$ is the minimum and $p_{\alpha}^{\max }$ is the maximum power that can be supplied by the battery, supercapacitor and FC.

\section{MLD constraints}

The ESS models represented in (1),(2) represents a hybrid model. In order to incorporate it in the optimisation problem they are converted into a mixed logical dynamic (MLD) system using boolean and auxiliary variable. The methodology outlined in [31] is used for the MLD formulation of the ESS models and is given by following set of equations

$$
\begin{aligned}
S O C_{\alpha}(i+1) & =S O C_{b}(i)+\frac{T_{s} \cdot}{C_{b}} \cdot z_{\alpha}(i) \cdot\left(\eta_{\alpha}-\frac{1}{\eta_{\alpha}}\right) \\
-\frac{T_{s} \cdot \eta_{\alpha}}{C_{b}} \cdot p_{\alpha}(i) & \\
-p_{\alpha}^{\min } \cdot \delta_{\alpha}(i) & \leq p_{\alpha}(i)-p_{\alpha}^{\text {min }} \\
-p_{\alpha}^{\max } \cdot \delta_{\alpha}(i) & \leq-p_{\alpha}(i) \\
z_{\alpha}(i) & \leq p_{\alpha}^{\text {max }} \cdot \delta_{\alpha}(i) \\
z_{\alpha}(i) & \geq p_{\alpha}^{\text {min }} \cdot \delta_{\alpha}(i) \\
z_{\alpha}(i) & \leq p_{\alpha}(i)+p_{\alpha}^{\text {max }} \cdot\left(1-\delta_{\alpha}(i)\right) \\
z_{\alpha}(i) & \geq p_{\alpha}(i)+p_{\alpha}^{\min } \cdot\left(1-\delta_{\alpha}(i)\right) \\
\forall \alpha & =\{b a t, s c\}
\end{aligned}
$$

where $\delta(i) \in\{0,1\}$ such that $[\delta(i)=1] \leftrightarrow\left[p_{\alpha} \geq 0\right]$ and $z_{\alpha}(i)=\delta(i) \cdot p_{\alpha}$ are the boolean and auxiliary variables respectively. The MLD formulation for the FC is same as (11) in terms of SOH. The above are linear constraints on mixed integer variable and can be solved using commercially available solvers.

Therefore the optimisation problem for the MPC can be summed up as follows

$$
J=\min \sum_{i=1}^{N-1}\left(J_{b a t}(i)+J_{f c}(i)+J_{s c}(i)+\rho \cdot J_{\text {slack }}\right)
$$

subject to

$$
\begin{array}{r}
\text { ESS model (1), (2) } \\
\text { Grid model (3) }
\end{array}
$$

Constraints (9), (10), (11)

where $\rho$ is penalty for slack variables of soft constraint.

\section{RESUlTS}

The system parameters used in the optimisation problem for energy management is shown Table.I. The proposed optimisation problem was solved using parser YALMIP and solver MOSEK (version 9.5) in MATLAB environment. It should also be noted that the simulations have been performed under the assumption that generation, load demand is deterministic and no uncertainty exists.

A logical solution to ensure efficient operation by energy management problem will be to provide a higher value for $c_{f c}$ on FC and lower value for $c_{b a t}$ as the cycling efficiency of the FC is lower than that of the battery. A straightforward choice will be using the inverse of cycle efficiency of FC and battery as the weights. A 60 and 90 percent cycle efficiency is considered for the FC and battery respectively [6]. This condition will be referred to as Case 1 and the values of 
ht

TABLE I

SYSTEM PARAMETERS AND THEIR VALUES USED

\begin{tabular}{|c|c|}
\hline Parameter & value \\
\hline$C_{s c}$ & $0.5 \mathrm{kWh}$ \\
\hline$C_{b a t}$ & $9 \mathrm{kwh}$ \\
\hline$C_{F C}$ & $6 \mathrm{kWh}$ \\
\hline Sampling time & $30 \mathrm{~min}$ \\
\hline MPC horizon $(\mathrm{N})$ & 10 \\
\hline$p_{b a t}^{\text {min }}, p_{b a t}^{\text {max }}$ & $2 \mathrm{~kW}$ \\
\hline$p_{s c}^{\text {min }}, p_{s c}^{\text {max }}$ & $2 \mathrm{~kW}$ \\
\hline$p_{f c}^{\text {max }}, p_{f c}^{\text {min }}$ & $2 \mathrm{~kW}$ \\
\hline$S O C_{b a t}^{l}, S O C_{s c}^{l}, S O H_{f c}^{l}$ & 0.2 \\
\hline$S O C_{b a t}^{u}, S O C_{s c}^{u}, S O H_{f c}^{u}$ & 0.95 \\
\hline$\left|p_{b a t \Delta}^{u}\right|,\left|p_{F C \Delta}\right|$ & $1 \mathrm{~kW}$ \\
\hline$S O C_{n o m}$ & 0.5 \\
\hline
\end{tabular}

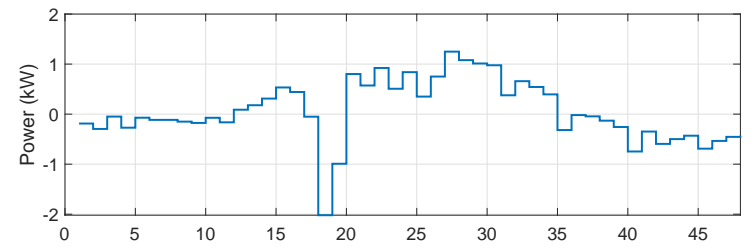

(a)

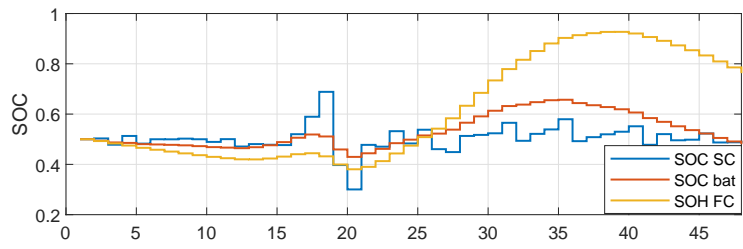

(b)

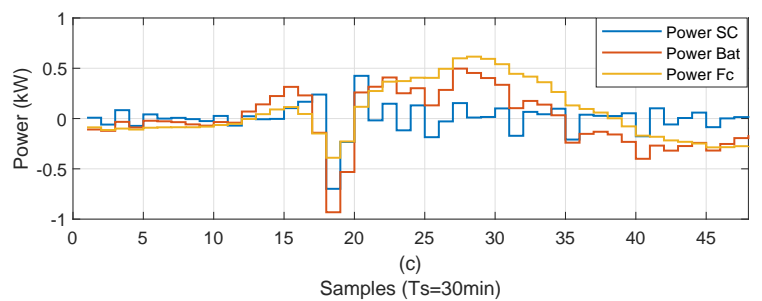

Fig. 3. Case 1:The results of energy management controller for (a) Imbalance power catered by ESS, (b) SOC, SOH evolution of ESS, (c) power supplied by the ESS

different penalty weights used in the optimisation problem is outlined in Table.II. The penalty weight $c_{s c}$ is kept at 2.5 to ensure that $S O C_{s c}$ is forced to remain around the $S O C_{n o m}$ which set at 0.5 to ensure that sufficient reserve exists in SC to absorb or provide imbalance power

The results from the energy management problem is shown in Fig.3. The Fig.3(a) shows the imbalance power $\left(p_{\text {gen }}-\right.$ $p_{\text {load }}$ ) in the grid catered by ESS. The Fig.3(b), (c) are the SOC evolution and power supplied by the different ESS. In order to quantify the performance of energy management controller
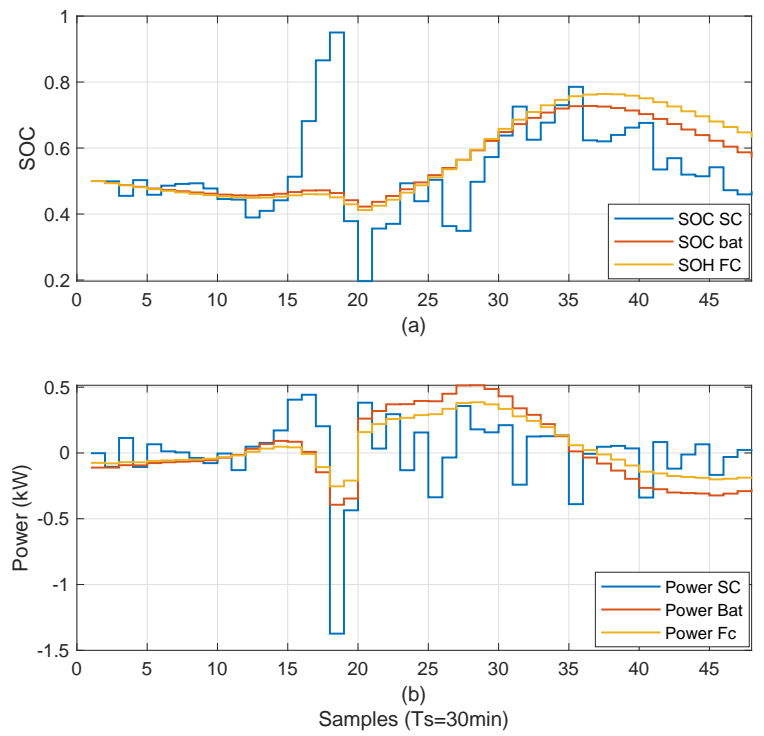

Fig. 4. Case 4: The results of energy management controller for (a) SOC, SOH evolution of ESS, (b) power supplied by the ESS

the following indices are proposed

$$
\begin{aligned}
c y c_{\alpha} & =\frac{\sum_{i=2}^{N-1}\left|S O C_{\alpha}(i+1)-S O C_{\alpha}(i)\right|}{2 \cdot\left(S O C \alpha^{u}-S O C \alpha^{l}\right)} \\
E H_{\alpha} & =c y c_{\alpha} \cdot C_{\alpha} \\
\forall \alpha & =b a t, f c
\end{aligned}
$$

where $c y c_{\alpha}$ indicates the number of cycles undergone by the ESS in one day with a value of 1 corresponding to one charge-discharge cycle given by SOC variation from $S O C \alpha^{l} \rightarrow S O C \alpha^{u} \rightarrow S O C \alpha^{l}$. This index is of interest as it is indicative of the degradation especially in the case of battery as higher cycling increases degradation rate [20]. The second index under consideration is $E H_{\alpha}$ which is the energy handled (stored and discharged) by the ESS. In an ESS with $1 \mathrm{kWh}$ storage capacity in one charge discharge cycle $E H_{\alpha}$ is 1 . This index is indicative of the efficiency of operation of the microgrid. If energy handled by ESS having low cycle efficiency like FC is high, provided battery is available for storage, it can lead to a less efficient operation.

The indices values for the Case 1 is provided in Table.II. It can be seen that energy handled by the ESS for this case is more or less equal and the cycle number for the battery is also low (0.3644). As can be seen from Fig.3b for the proposed weights $S O C_{s c}$ is maintained close to 0.5 allowing it to cater unforeseen surplus or deficiency in demand. This looks like an acceptable operation condition nevertheless it needs to be explored whether at different penalty weight values an improved performance can be achieved.

In this context simulation were carried out for different cases emphasising different weights and important scenarios along with performance indices are summarised in Table.II. 
TABLE II

SIMULATION RESULTS FOR DIFFERENT CASES AND THEIR PERFORMANCE INDICES

\begin{tabular}{|c|c|c|c|c|c|c|c|c|c|c|}
\hline \multirow{2}{*}{ Case } & \multicolumn{7}{|c|}{ Parameters } & \multicolumn{4}{c|}{ Results } \\
\cline { 2 - 11 } & $c_{\text {bat }}$ & $c_{\text {soc }}$ & $c_{\text {dod }}$ & $c_{f c}$ & $c_{\text {rate }}$ & $c_{s c}$ & $c y c_{\text {bat }}$ & $E H_{\text {bat }}$ & $c y c_{f c}$ & $E H_{f c}$ \\
\hline 1 & 1.1 & 1 & 1 & 1.67 & 1 & 2.5 & 0.3644 & 3.2794 & 0.5495 & 3.2971 \\
\hline 2 & 1.1 & 1 & 1 & 10 & 1 & 2.5 & 0.6319 & 5.6872 & 0.1147 & 0.68835 \\
\hline 3 & 10 & 1 & 1 & 1.667 & 1 & 2.5 & 0.1328 & 1.195 & 0.7839 & 4.7034 \\
\hline 4 & 10 & 1 & 1 & 10 & 1 & 2.5 & 0.3566 & 3.2098 & 0.3712 & 2.2273 \\
\hline 5 & 1.1 & 10 & 10 & 10 & 1 & 2.5 & 0.4358 & 3.922 & 0.4027 & 2.416 \\
\hline 6 & 1.1 & 10 & 10 & 1.667 & 1 & 2.5 & 0.2679 & 2.4108 & 0.6810 & 3.845 \\
\hline 7 & 1.1 & 1 & 1 & 1.667 & 10 & 2.5 & 0.4259 & 3.8340 & 0.4549 & 2.7291 \\
\hline
\end{tabular}

It should be noted in all the cases penalty weights for the slack variables are kept at 500 . The worst performers have been Case 2 and 3. This is mainly due to excessive battery cycling with minimum FC energy handling in case 2 leading to increased battery degradation and vice-versa in case 3 resulting in inefficient performance as seen from the indices values. Therefore excessive emphasis of only $c_{b a t}$ or $c_{f c}$ is not advisable. Another ad performer is case 6 where $c_{s o c}, c_{d o d}$ are emphasised which leads to higher energy handling of FC. Nevertheless in case 6 it still does not lead to very high values of $E H_{f c}$ as in case 3. Therefore it can be concluded that emphasising $c_{b a t}$ has a more profound impact than $c_{s o c}, c_{d o d}$.

Case 4 appears to provide a good performance with low $c y c_{b a t}$ and low $c_{f c}$ nonetheless it can be misleading. The low indices value means an increased utilisation of the SC as can be seen from Fig.4. It can be noticed from Fig.4 that between sampling instance 15 and 25 the SC tends to be highly charged or deep discharged which reduces its ability to cater to unforeseen load variation thereby affecting the voltage regulation performance. Therefore providing high weights on both $c_{b a t}$ and $c_{f c}$ is best avoided to minimise the increased utilisation SC.

Finally case 5 and 7 provide good performance with both maintaining low $c y c_{b a t}$ while keeping the $E H_{f c}$ low and lesser than $E H_{b a t}$ which is indicative of more efficient performance in comparison to case 1 (as $E H_{b a t}$ and $E H_{f c}$ are more or less equal). The case 5 and 7 performance are shown in Fig.5 and Fig. 6 respectively. Case 5 compared to 7 provides some instances of deep discharge in SC, as can be seen at 18th sample, which can make it less desirable from the perspective of SC utilisation. In comparison case 7 with an increased emphasis on the rate of change of FC set point ensures better protection for the FC.

In order to summarise case 1 and 7 provide good performance indices while keeping SC utilisation also within limits. The selection criteria depends on the priority placed on the grid operation whether to have a more efficient or less degrading one. These tend to be complementary and as such suitable selection needs to be taken based on operator requirements.

The Fg.7 shows MPC based energy management scheme integrated with the low level control for voltage regulation in the grid. The low level control corresponds to the short term power balancing scheme discussed in the introduction. As can be seen from Fig.7 the generated set points from
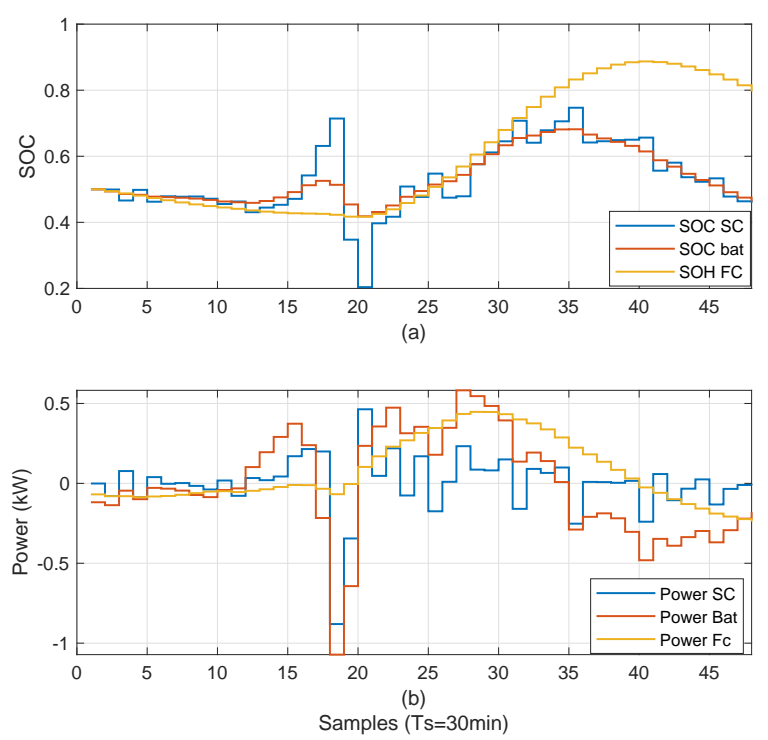

Fig. 5. Case 5: The results of energy management controller for (a) SOC, SOH evolution of ESS, (b) power supplied by the ESS
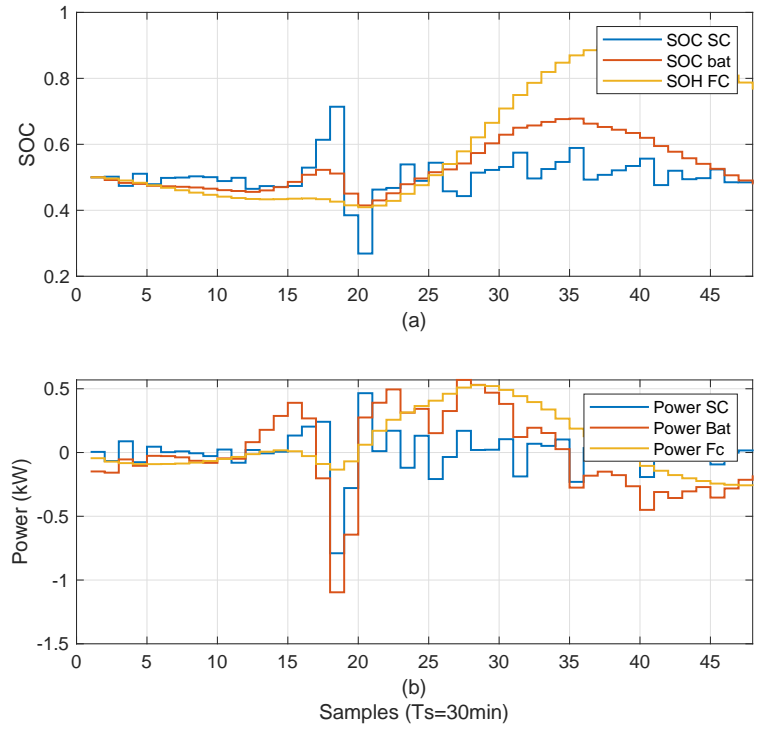

Fig. 6. Case 7: The results of energy management controller for (a) SOC, SOH evolution of ESS, (b) power supplied by the ESS 


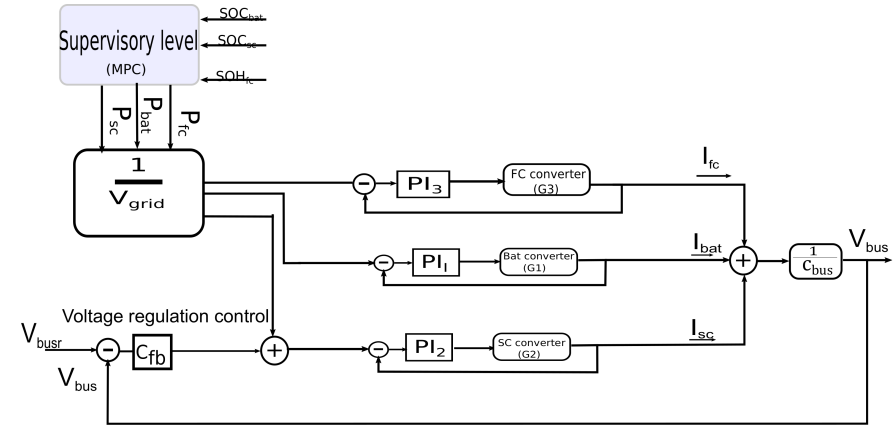

Fig. 7. The low level control schematic integrated with MPC based intraday energy management scheme

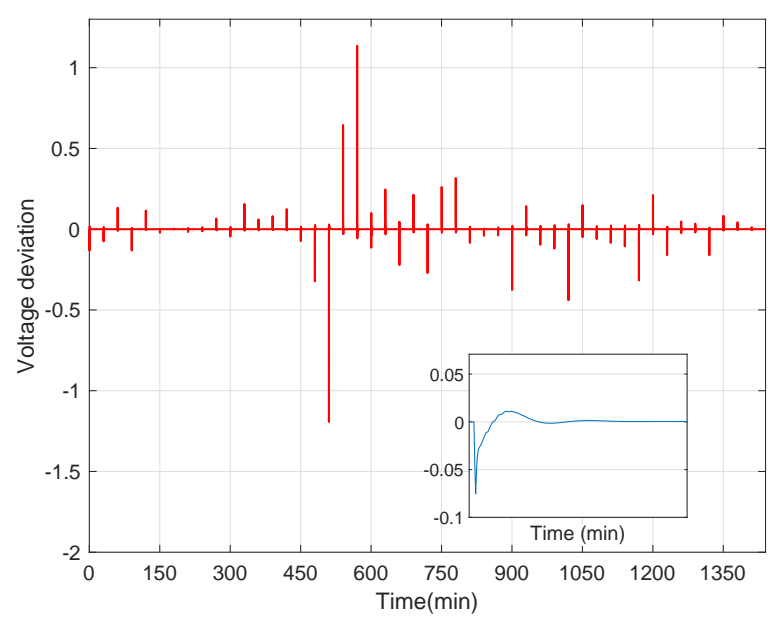

Fig. 8. The voltage regulation performance by low level controller aided by energy management algorithm with dynamic response in the inset

MPC $\left(P_{f c}, P_{b a t}, P_{s c}\right)$ is applied to the current controllers of the FC, battery and SC power converters which will ensure that the required current is injected to the grid to ensure stability and voltage regulation. The SC converter is also provided with a cascaded control scheme with an outer voltage control loop for regulation. This takes care of maintaining the grid voltage within prescribed limits under unforeseen disturbances. The MPC set point for SC will be augmented with the voltage control loop output. The Fig.8 shows voltage regulation performance of low level controllers when working in tandem with MPC. The results were obtained using cosimulation by simultaneously running a MATLAB code along with a Simulink model where the MPC and low level control schemes were implemented respectively. The MATLAB code sampled the data from Simulink at every sampling instant and used it for solving the optimisation problem. The case 7 parameters are used in the optimisation. The voltage regulation transient response is shown in the inset of Fig.8 and is dominated by the SC control loop dynamics. The unmodelled delays of the low level do not have any relevant impact on the energy management algorithm performance and its ability to find an optimal solution.

\section{CONCLUSION}

An intraday energy management scheme has been proposed in this work which aims at minimising degradation rate and optimise operating efficiency of microgrid. In this context the work proposed cost function which are quadratic in nature that can heuristically reduce the degradation rate along with some performance indices which allowed to quantify the grid performance. The interaction of ESS based on weighting factors were studied through different cases. The case enabled to develop a general consensus regarding the selection of penalty weights in optimisation problem.

A major observation from this work is that objectives in the optimisation problem and resulting ESS interaction tend to be complementary. This follows that while degradation rate is reduced the operational efficiency tend to low and vice versa, also while battery, FC utilisation is reduced the SC utilisation increases which can reduces the reserve capacity of the grid to cater unforeseen disturbance variation. This allows use of multi objective optimisation techniques including Pareto optimisation for improved decision making in future work. Another aspect that future work can focus on is performance of the MPC energy management controller under a non-deterministic generation. In this context investigation of stochastic MPC techniques can be carried out. Apart from this the low level controller interaction with MPC controller under stochastic generation, load profiles can be carried out as well.

\section{REFERENCES}

[1] M Stanley Whittingham. History, evolution, and future status of energy storage. Proceedings of the IEEE, 100(Special Centennial Issue):15181534, 2012.

[2] Marcelo G Molina. Energy storage and power electronics technologies: A strong combination to empower the transformation to the smart grid. Proceedings of the IEEE, 105(11):2191-2219, 2017.

[3] Josep M Guerrero, Juan C Vasquez, José Matas, Luis García De Vicuña, and Miguel Castilla. Hierarchical control of droop-controlled ac and dc microgridsa general approach toward standardization. IEEE Transactions on industrial electronics, 58(1):158-172, 2011.

[4] Daniel E Olivares, Ali Mehrizi-Sani, Amir H Etemadi, Claudio A Cañizares, Reza Iravani, Mehrdad Kazerani, Amir H Hajimiragha, Oriol Gomis-Bellmunt, Maryam Saeedifard, Rodrigo Palma-Behnke, et al. Trends in microgrid control. IEEE Transactions on smart grid, 5(4):1905-1919, 2014.

[5] Ali Bidram and Ali Davoudi. Hierarchical structure of microgrids control system. IEEE Transactions on Smart Grid, 3(4):1963-1976, 2012.

[6] Haisheng Chen, Thang Ngoc Cong, Wei Yang, Chunqing Tan, Yongliang $\mathrm{Li}$, and Yulong Ding. Progress in electrical energy storage system: A critical review. Progress in Natural Science, 19(3):291 - 312, 2009.

[7] Paul Denholm, Erik Ela, Brendan Kirby, and Michael Milligan. The role of energy storage with renewable electricity generation. 2010.

[8] Erik Schaltz, Alireza Khaligh, and Peter Omand Rasmussen. Influence of battery/ultracapacitor energy-storage sizing on battery lifetime in a fuel cell hybrid electric vehicle. IEEE Transactions on Vehicular Technology, 58(8):3882-3891, 2009.

[9] H. Kanchev, D. Lu, F. Colas, V. Lazarov, and B. Francois. Energy management and operational planning of a microgrid with a pv-based active generator for smart grid applications. IEEE Transactions on Industrial Electronics, 58(10):4583-4592, Oct 2011.

[10] Pablo Garcia, Luis M Fernandez, Carlos Andres Garcia, and Francisco Jurado. Energy management system of fuel-cell-battery hybrid tramway. IEEE Transactions on Industrial Electronics, 57(12):4013-4023, 2010.

[11] MC Kisacikoglu, M Uzunoglu, and MS Alam. Load sharing using fuzzy logic control in a fuel cell/ultracapacitor hybrid vehicle. International journal of hydrogen energy, 34(3):1497-1507, 2009. 
[12] Dennice Gayme and Ufuk Topcu. Optimal power flow with large-scale storage integration. IEEE Transactions on Power Systems, 28(2):709_ 717, 2013.

[13] Tu A Nguyen and ML Crow. Stochastic optimization of renewablebased microgrid operation incorporating battery operating cost. IEEE Transactions on Power Systems, 31(3):2289-2296, 2016.

[14] Rodolfo Dufo-Lopez, José L Bernal-Agustín, and Javier Contreras. Optimization of control strategies for stand-alone renewable energy systems with hydrogen storage. Renewable energy, 32(7):1102-1126, 2007.

[15] Felix Garcia-Torres, Luis Valverde, and Carlos Bordons. Optimal load sharing of hydrogen-based microgrids with hybrid storage using model-predictive control. IEEE Transactions on Industrial Electronics, 63(8):4919-4928, 2016

[16] M Khalid and AV Savkin. A model predictive control approach to the problem of wind power smoothing with controlled battery storage. Renewable Energy, 35(7):1520-1526, 2010.

[17] A. Parisio, E. Rikos, and L. Glielmo. A model predictive control approach to microgrid operation optimization. IEEE Transactions on Control Systems Technology, 22(5):1813-1827, Sep. 2014.

[18] Duong Tran and Ashwin M Khambadkone. Energy management for lifetime extension of energy storage system in micro-grid applications. IEEE Transactions on Smart Grid, 4(3):1289-1296, 2013.

[19] Austin Hausmann and Christopher Depcik. Expanding the peukert equation for battery capacity modeling through inclusion of a temperature dependency. Journal of Power Sources, 235:148-158, 2013.

[20] John Wang, Ping Liu, Jocelyn Hicks-Garner, Elena Sherman, Souren Soukiazian, Mark Verbrugge, Harshad Tataria, James Musser, and Peter Finamore. Cycle-life model for graphite-lifepo4 cells. Journal of Power Sources, 196(8):3942-3948, 2011.

[21] James Blake Rawlings and David Q Mayne. Model predictive control: Theory and design. Nob Hill Pub. Madison, Wisconsin, 2009.
[22] Anthony Barré, Benjamin Deguilhem, Sébastien Grolleau, Mathias Gérard, Frédéric Suard, and Delphine Riu. A review on lithium-ion battery ageing mechanisms and estimations for automotive applications. Journal of Power Sources, 241:680-689, 2013.

[23] J Vetter, Petr Novák, Markus Robert Wagner, Claudia Veit, K-C Möller, JO Besenhard, Martin Winter, Margret Wohlfahrt-Mehrens, C Vogler, and A Hammouche. Ageing mechanisms in lithium-ion batteries. Journal of power sources, 147(1-2):269-281, 2005.

[24] Gang Ning, Bala Haran, and Branko N Popov. Capacity fade study of lithium-ion batteries cycled at high discharge rates. Journal of Power Sources, 117(1-2):160-169, 2003.

[25] Akira Taniguchi, Tomoki Akita, Kazuaki Yasuda, and Yoshinori Miyazaki. Analysis of electrocatalyst degradation in pemfc caused by cell reversal during fuel starvation. Journal of Power Sources, 130(12):42-49, 2004.

[26] Timothy W Patterson and Robert M Darling. Damage to the cathode catalyst of a pem fuel cell caused by localized fuel starvation. Electrochemical and Solid-State Letters, 9(4):A183-A185, 2006.

[27] N Yousfi-Steiner, Ph Moçotéguy, D Candusso, and D Hissel. A review on polymer electrolyte membrane fuel cell catalyst degradation and starvation issues: Causes, consequences and diagnostic for mitigation. Journal of Power Sources, 194(1):130-145, 2009.

[28] P. Thounthong and P. Sethakul. Analysis of a fuel starvation phenomenon of a pem fuel cell. In 2007 Power Conversion Conference - Nagoya, pages 731-738, April 2007.

[29] R Kötz and M Carlen. Principles and applications of electrochemical capacitors. Electrochimica acta, 45(15-16):2483-2498, 2000.

[30] Eric C Kerrigan and Jan M Maciejowski. Soft constraints and exact penalty functions in model predictive control. 2000.

[31] Alberto Bemporad and Manfred Morari. Control of systems integrating logic, dynamics, and constraints. Automatica, 35(3):407-427, 1999. 